\title{
Psychological adjustment among partners of women at high risk of developing breast/ovarian cancer
}

Shab Mireskandari, DPsych ${ }^{1,2,3}$, Kerry A. Sherman, PhD ${ }^{1}$, Bettina Meiser, PhD ${ }^{2,4}$, Alan J. Taylor, PhD ${ }^{1}$, Margaret Gleeson, BAppSci, Grad Dip Genet Couns ${ }^{5}$, Lesley Andrews, MBBS DipObs, MMed ${ }^{6}$, and Katherine M. Tucker, FRACP 3,6

\begin{abstract}
Purpose: The aim of the current research was to characterize psychological adjustment among partners of women at high risk of developing breast/ovarian cancer and to explore the relationship between women's and partners' adjustment. Methods: A study of 95 unaffected at-risk women and 95 partners was carried out using mailed, self-administered questionnaires with validated measures of psychological outcome. Results: Elevated levels of distress were noted in up to $10 \%$ of partners. High monitoring coping style and greater perceived breast cancer risk for their wife were associated with higher distress levels for partners. However, communicating openly with their wife and the occurrence of a recent cancer-related event in the woman's family were related to lower distress for partners. Partners' cancer-specific distress was positively related to their wives' distress. Conclusion: Among partners with elevated levels of distress, the ability to provide effective support to the at-risk women and participate appropriately in their decision making may be compromised. These partners are likely to benefit from targeted clinical interventions designed to reduce their distress levels. The findings emphasize the importance of considering partners of at-risk women in service provision and highlight the need for partners to obtain information and support specifically tailored to their needs. Genet Med 2007:9(5):311-320.
\end{abstract}

Key Words: hereditary breast/ovarian cancer, partners, psychological adjustment, cognitive-social health information processing (C-SHIP) model, couples

Approximately $5 \%$ of all breast/ovarian cancers are considered to be attributable to a dominantly inherited breast/ovarian cancer predisposition. ${ }^{1}$ In the past decade, two breast/ovarian cancer predisposition genes, BRCA1 and BRCA2, have been identified, with the risk of developing cancer imparted by mutations in these genes estimated to be between $45 \%$ and $65 \%$ for breast cancer and between $10 \%$ and $40 \%$ for ovarian cancer by age 70 years. ${ }^{1}$ Women who are at increased risk of hereditary breast/ovarian cancer, and in particular those found to be mutation carriers, need to make decisions about how to best manage their risk. Women may opt for frequent screening, prophylactic mastectomy and/or oophorectomy, ${ }^{2}$ and/or chemoprevention. Given that having a strong family history of breast/ovarian cancer may result in women experiencing ele-

From the ${ }^{1}$ Department of Psychology, Macquarie University, Sydney, Australia; ${ }^{2}$ Psychosocial Research Group, Department of Medical Oncology, Prince of Wales Hospital, Sydney, Australia; ${ }^{3}$ Prince of Wales Clinical School, University of NSW, Sydney, Australia; ${ }^{4}$ School of Psychiatry, University of NSW, Sydney, Australia; ${ }^{5}$ Hunter Family Cancer Service, Hunter Genetics, Newcastle, Australia; ${ }^{6}$ Hereditary Cancer Clinic, Prince of Wales Hospital, Sydney, Australia.

Dr. Shab Mireskandari, Psychosocial Research Group, Dickinson 3, Prince of Wales Hospital, Randwick, NSW 2031, Australia; E-mail: shab.mireskandari@psy.mq.edu.au

The authors declare no conflicts of interest.

Submitted for publication December 5, 2006.

Accepted for publication February 26, 2007.

DOI: 10.1097/GIM.0b013e3180534293 vated levels of cancer-related distress, ${ }^{3}$ social support from the women's families and significant others, particularly their partners, has been identified as a key resource in enabling the women to effectively cope with their increased cancer risk. ${ }^{4,5}$

Few studies have explicitly focused on the adjustment of partners of women at high risk of developing breast/ovarian cancer. ${ }^{2,5-11}$ Investigations of the psychological impact of genetic testing on partners of at-risk women have revealed that the genetic testing process and, in particular, women found to be mutation carriers can be distressing for some partners. ${ }^{2,7-9}$ Men who felt understood by their wives and felt more comfortable sharing their concerns about genetic testing with the women at the pretest education time reported lower distress at 6-month follow-up. ${ }^{5}$ Research with partners of unaffected women at high risk who have undergone prophylactic bilateral mastectomy suggests that after the operation, the partners were subject to stress in their supportive role, balancing the multiple demands of emotional and practical support, continuing work, and child care. ${ }^{10}$

The primary aim of this study was to characterize psychological adjustment among partners of women at high risk of developing breast/ovarian cancer using a survey design. Specifically, guided by the cognitive-social health information processing (C-SHIP) model, ${ }^{12}$ this study seeks to delineate the individual (partners' personal characteristics) and couple (characteristics of the partners' relationship with the at-risk 
women) psychological factors associated with partners' adjustment. The C-SHIP model includes two basic components: a set of mediating cognitive-affective units and the structure and dynamic processes through which these units interact to generate health-protective behavior. ${ }^{12}$ Three of the relevant mediating units hypothesized by the C-SHIP model are health-relevant encodings (e.g., risk perception), health beliefs and expectancies (e.g., likelihood of developing cancer and expectancies about outcome), and self-regulatory competencies and skills (e.g., anxiety management ability). ${ }^{12}$ Based on this model, the impact of women's high risk of breast/ovarian cancer on their partners would depend, in part, on how the women's risk is interpreted, and the beliefs, expectancies, and selfregulatory skills that are activated for partners during the processing of this information. The model predicts that better adjustment (lower general and cancer-specific distress) for partners would be associated with (i) lower perceived risk of breast/ovarian cancer, (ii) greater positive beliefs about the onset and management of breast/ovarian cancer, and (iii) greater self-regulatory ability. It further predicts that the effects of the mediating units will be moderated by attentional style, with individuals with a tendency to monitor for threatening information (high monitors) likely to experience greater perceived risk than low monitors. ${ }^{12}$ In addition, our previous qualitative investigations ${ }^{11}$ identified possible couple factors associated with partners' adjustment, and, based on these findings, it is predicted that better adjustment (lower general and cancerspecific distress) for partners will be associated with (i) feeling adequate in providing support to their wives, (ii) greater involvement in decision making, (iii) the perception that their wives are coping well, (iv) agreement between the woman and her partner about cancer-related issues, including satisfaction with the management of her risk, (v) having a team approach, and (vi) communicating openly about this issue. A secondary aim of this study is to explore the association between partners' and women's adjustment. Because research with women with breast cancer and their partners has consistently shown a positive correlation between women's and partner's adjustment, ${ }^{13-15} \mathrm{a}$ similar positive association is also likely for at-risk women's and their partners' adjustment. Furthermore, this study explored partners' information and support needs, concerns regarding children, and the partners' views regarding the impact on their relationships.

\section{METHODS}

\section{Participants and data collection}

To recruit partners of women at high risk of developing breast/ovarian cancer, the medical records of two major familial cancer clinics in New South Wales, Australia, were searched to identify women who had attended these clinics for face-toface genetic counseling between July 2000 and February 2004. These clinics provide risk assessment, genetic testing where appropriate, and advice regarding early detection and prophylactic strategies according to national guidelines. ${ }^{16}$ Currently, only women at risk of developing hereditary breast/ovarian cancer who have an affected living relative willing to provide a blood sample are eligible for genetic testing (in only 20\%-25\% of families suggestive of hereditary breast/ovarian cancer) because the test is informative only when a known mutation is segregating in a particular family. ${ }^{17}$ To be eligible for the current study, women had to have no history of breast/ovarian cancer, be 18 years of age and older, fluent in English (because data were collected using self-administered questionnaires), have a family history consistent with dominantly inherited breast/ovarian cancer, and a mutation carrier risk of at least $25 \%$ (corresponding to a lifetime risk of $25 \%-80 \%$ ). ${ }^{18}$ An approximate $25 \%$ mutation carrier risk applies to a woman from a high-risk family whose closest affected relative is a seconddegree relative or a woman from a family with an identified mutation whose closest relative with a mutation is a seconddegree relative. Known noncarriers of BRCA1/2 mutations and those with a lower lifetime risk were excluded from participation.

All eligible women were contacted by letter by the treating clinician (K.T., L.A., M.G.). Women were asked to return an opt-out card or call their clinic if they did not have a partner/ husband or if they and/or their partners did not wish to participate in the study. Women who did not return their opt-out cards or did not call to opt out received a recruitment telephone call ascertaining their decision about the study. Women who agreed for themselves and their partners to be included in the study were sent a package including separate questionnaires, information and consent forms, and reply paid envelopes for the women and their partners. Women and partners were given specific instructions not to assist each other with the answers while completing their questionnaires.

\section{Measures}

The following measures were administered in separate questionnaires to both women and their partners. The scope of data collected from the couples in this sample exceeds data that were used for the purposes of this study and only the subset of the collected data relevant to the aim of the current study is reported below (i.e., all information collected from partners is reported and analyzed, and only demographic data, scores on the outcome measures, and relevant predictor variables are reported and analyzed for women). The remaining data will be reported elsewhere.

\section{Predictor variables}

\section{Demographic characteristics}

Information regarding age, educational level, marital status, relationship length, and number of biological children were collected.

\section{Family history and genetic testing data}

Data on the number of first- and second-degree relatives with breast and/or ovarian cancer, genetic testing status and time since the women had attended genetic counseling were collected from medical records. 


\section{Miller behavioral style scale}

This four-item scale is a validated measure of monitoring attentional style. ${ }^{19}$ It measures responses to four hypothetical stress-invoking scenarios. Each scenario is followed by eight statements that represent different strategies for dealing with the stressful event, with four responses indicative of a high monitoring style, and four indicative of a low monitoring style. In the present study, only the sum of all the high monitoring response options endorsed across the four scenarios (i.e., the monitoring score) is used, as the monitoring dimension has been found to be a better predictor of adjustment. ${ }^{20}$ Based on the C-SHIP model, monitoring is included in the present study as a moderating variable in the analyses.

\section{Breast/ovarian cancer genetics knowledge scale}

This eight-item measure adapted from a scale used in previous breast cancer genetics research ${ }^{21}$ assesses knowledge about breast/ovarian cancer genetics and was included because knowledge has been related to breast/ovarian cancer-specific anxiety. One point is given for each correct answer and a total score is calculated (range, $0-8$ ). This scale has been found to have adequate internal consistency with $\alpha=0.59 .{ }^{21}$

\section{Individual and couple factors}

Based on the C-SHIP model, nine items were designed to assess the three individual cognitive-affective mediating units, whereas nine items were specifically designed to assess the couple psychological factors derived from our previous qualitative research. ${ }^{11}$ A detailed description of these items is provided below (see Results).

\section{Breast/ovarian cancer-related event in the family}

A single item asked women whether they had experienced any breast/ovarian cancer-related event such as mother's or sister's diagnosis or death from breast or ovarian cancer in the past year. Breast/ovarian cancer-related events in the family were assessed because such events may act as psychological risk factors. ${ }^{22} \mathrm{~A}$ time frame of 12 months was chosen for the assessment of breast/ovarian cancer-related life events, as previous studies show that among affected women from hereditary breast cancer families, those diagnosed less than 1 year ago had significantly elevated distress compared with women diagnosed more than 1 year ago, ${ }^{23}$ suggesting that a 12 -month time frame is meaningful within this context. To avoid duplication, this item was not administered to partners.

\section{Outcome variables}

\section{General distress}

General distress was measured using the Depression, Anxiety, and Stress Scale-Brief version (DASS). ${ }^{24}$ This 21 -item scale is a validated measure of general depression, anxiety, and stress for which Australian population norms are available. ${ }^{24}$ Subjects used a 4-point severity/frequency scale to rate the extent to which they experienced each state over the past week, ranging from "did not apply to me at all" to "applied to me very much or most of the time." 24 Scores for the subscales range from 0 to 42 . Cronbach's $\alpha$ values for the three subscales in the present sample indicated high internal consistency: depression, 0.81 ; anxiety, 0.73 ; and stress, $0.81 .{ }^{24}$

\section{Cancer-specific distress}

The Impact of Event Scale (IES) ${ }^{25}$ was used to measure cancer-specific distress. This 15 -item scale is a validated measure of intrusion (seven-item subscale) and avoidance (eight-item subscale) responses in relation to a specific stressor, ${ }^{25}$ which in the present study was concern about women being at increased risk of developing breast/ovarian cancer. Participants rated symptoms of distress over the past week on a scale ranging from "not at all" to "often." Both the intrusion and avoidance subscales demonstrate high internal consistency, with Cronbach's $\alpha=0.78$ and 0.82 , respectively. ${ }^{25}$ An $\alpha$ value of 0.87 was obtained for the intrusion and 0.86 for the avoidance subscales for partners in the present study.

\section{Information and support needs}

Ten items (five open-ended questions and five multiple choice questions with options ranging from "strongly disagree" (1) to "strongly agree" (5) were specifically designed to assess partners' information and support needs, concerns for children also being at increased risk, views regarding cancer risk management options for women, and the impact of the women's cancer risk on the relationship.

\section{Data analysis}

Data were analyzed using SPSS 12.0 (Statistical Program for the Social Sciences). Descriptive statistics were used to summarize participant characteristics, including psychological status. Initially, bivariate analyses exploring associations between the partners' predictor variables and potentially confounding variables (such as partners' age, educational level, and length of relationship) and the two outcome variables (partners' cancer-related distress as measured by partners' IES scores, and partners' general distress as measured by the DASS) were undertaken using the nonparametric statistical tests of Spearman's rank correlations (for continuous variables) and Mann-Whitney $U$ test (for categorical variables). As both outcome variables were highly negatively skewed, in the multivariate analyses, each of the outcome variables was recoded into an ordinal categorical variable with three levels (one level including all cases with a score of zero, and the other two levels splitting the remaining cases into two approximately equal groups). Also, total IES and DASS scores were used in the multivariable analyses because similar results were obtained using the IES and DASS subscales (correlation for IES subscales $=0.72$ and for DASS subscales range $=0.67-0.77)$. Predictor variables identified as having a $P \leq 0.1$ in the bivariate analyses were included in a full ordinal logistic regression model for the DASS and a multinominal logistic regression for the IES. The full regression models were then reduced, one predictor variable at a time, with the variable with the highest $P$ value removed at each step, until final models, which contained only variables with $P<0.05$, were ob- 
tained. To explore the association between partners' adjustment and women's adjustment, Spearman's rank correlations were calculated between partners' and women's IES and DASS scores, and to compare partners' and women's scores on each of the relevant measures, the Wilcoxon test was used for scores with a skewed distribution and a paired $t$ test was used for normally distributed scores.

\section{RESULTS}

\section{Demographics}

Letters of invitation were sent out to 218 women. Of these, 30 were lost to contact. Of the remaining 188 women, 37 women were not eligible to participate in the study: two were non-English speaking, one had recently been found not to be a mutation carrier, six had developed breast or ovarian cancer since their last visit to the clinic, and 28 did not have a partner/ husband. Of the remaining 151 eligible women, 28 women, two partners and two couples opted out of the study, $16 \mathrm{cou}-$ ples did not return their questionnaires, and eight couples withdrew from the study. Hence, data were collected from a total of 95 couples ( 95 women and 95 partners), resulting in a participation rate of $62.9 \%$.

Sociodemographic, family history, and genetic testing characteristics of the participants are summarized in Table 1. The mean time since the women had attended genetic counseling was 21.8 months $(\mathrm{SD}=20.0$; range $=1-94$ months $)$. The sample included $13(13.7 \%)$ women who were BRCA1/2 mutation carriers and $82(86.3 \%)$ women with unknown mutation status; most women in this latter group were not eligible for testing because no family-specific mutation had been identified. The average age of the women was 42.9 years $(S D=9.4$; range $=23-67$ years). All partners were male with an average age of 45.4 years $(S D=10.2$; range $=23-72$ years). The majority of the couples were married $(90.5 \%)$, with the remaining couples $(9.5 \%)$ in a committed relationship and living together; the average relationship length was 18.3 years $(S D=11.2$; range $=1-46$ years). Most couples $(80.0 \%)$ had biological children with their current partner or were currently pregnant with their first child.

\section{Predictor variables associated with partners' adjustment}

All individual and couple factor items, including response options, means, and SDs, are shown in Table 2. While a single item represented several of the individual and couple factors, three factors were combined items as shown in Table 2 (see brackets next to items), on the basis of exploratory/confirmatory factor analyses: mean fatalism ( mean $=1.85 ; \mathrm{SD}=0.82$ ), mean self-regulatory abilities ( mean $=1.85 ; \mathrm{SD}=0.82)$, and mean supportive role $($ mean $=1.85 ; \mathrm{SD}=0.82)$. Mean scores were obtained for these factors as the combined items were correlated (range $=0.33-0.70)$ and had $\alpha$ reliability of 0.70 or higher (range $=0.73-0.76$ ). Exploratory and confirmatory factor analyses confirmed satisfactory factor loadings for each of the four scales comprised of combined items, with factor loadings ranging from 0.65 to 0.78 .
Table 1

Summary of participant characteristics

\begin{tabular}{lcc}
\hline Variable & $\begin{array}{c}\text { Partner } \\
(N=95)\end{array}$ & $\begin{array}{c}\text { Woman } \\
(N=95)\end{array}$ \\
\hline Age $(y r)$ & & \\
Mean (SD) & $45.4(10.2)$ & $42.9(9.4)$ \\
Range & $23-72$ & $23-67$ \\
Education & & \\
No postschool qualifications & $33(34.7 \%)$ & $26(27.4 \%)$ \\
Postschool qualifications & $62(65.3 \%)$ & $69(72.6 \%)$ \\
Woman's no. of FDRs and SDRs ${ }^{a}$ & & \\
$1-2$ & & $47(51.7 . \%)$ \\
$3-4$ & & $39(42.9 \%)$ \\
$5-6$ & & $5(5.5 \%)$
\end{tabular}

Woman's risk status

Unknown mutation status

Carrier

$13(13.7 \%)$

Woman's prophylactic surgery status

No surgery

$82(86.3 \%)$

Bilateral prophylactic mastectomy

$2(2.1 \%)$

Bilateral prophylactic oophorectomy

$9(9.5 \%)$

Prophylactic mastectomy and oophorectomy

$2(2.1 \%)$

Relationship status

Married

$86(90.5 \%)$

De facto

$9(9.5 \%)$

Relationship length $($ mean $=18.3 \mathrm{yr}$; range $=1-46 \mathrm{yr}$ )

1-9

$20(21.0 \%)$

10-19

$34(35.8 \%)$

20-29

$22(23.2 \%)$

$>30$

$19(20.0 \%)$

Children (mean no. of children with current partner 2 ; range $=0-5$ )

No children with current partner

$19(20.0 \%)$

Children with current partner or pregnant

$76(80.0 \%)$

At least one female offspring

$52(68.4 \%)^{b}$

No female offspring

$23(30.3 \%)^{b}$

Pregnant with first child

$1(1.3 \%)^{b}$

Language spoken at home

English $\quad 82(86.3 \%)$

Other

$13(13.7 \%)$

$\overline{{ }^{a} \text { Wife's total number of first- and second-degree relatives with breast and/or }}$ ovarian cancer.

${ }^{b}$ Percentages calculated for couples who had children with current partner (i.e., $N=76$ ). 
Table 2

Individual and couple psychological factors

\begin{tabular}{|c|c|c|c|c|}
\hline Factor & Item & Mean & SD & $\%$ \\
\hline \multicolumn{5}{|l|}{ Individual factors } \\
\hline \multirow[t]{2}{*}{$\begin{array}{l}\text { Perceived risk of developing } \\
\text { breast/ovarian cancer }\end{array}$} & $\begin{array}{l}\text { What are your partner's chances of developing breast } \\
\text { cancer compared with other women her age? }\end{array}$ & 4.14 & 0.81 & $84.2^{a}$ \\
\hline & $\begin{array}{l}\text { What are your partner's chances of developing ovarian } \\
\text { cancer compared with other women her age? }\end{array}$ & 3.35 & 1.00 & $42.1^{a}$ \\
\hline \multirow{5}{*}{$\begin{array}{l}\text { Beliefs and expectancies about } \\
\text { cancer } 1\end{array}$} & I believe screening will help in detecting cancer early & 4.00 & 0.73 & $83.2^{b}$ \\
\hline & $\begin{array}{l}\text { I believe prophylactic surgery is effective in preventing } \\
\text { breast/ovarian cancer }\end{array}$ & 3.35 & 1.04 & $48.4^{b}$ \\
\hline & $\begin{array}{l}\text { I believe cancer is fatal, even if the cancer is detected early } \\
\text { due to regular screening }\end{array}$ & 2.06 & 1.00 & $11.6^{c}$ \\
\hline & $\begin{array}{l}\text { I believe that if someone has cancer, it is already too late to } \\
\text { do anything about it }\end{array}$ & 1.63 & 0.84 & $4.2^{c}$ \\
\hline & $\begin{array}{l}\text { I am optimistic that my partner will not develop breast/ } \\
\text { ovarian cancer }\end{array}$ & 3.67 & 0.95 & $61.1^{c}$ \\
\hline \multirow[t]{2}{*}{ Self-regulatory strategies 2} & $\begin{array}{l}\text { I am able to limit the amount of stress I experience about } \\
\text { my partner's increased cancer risk }\end{array}$ & 3.54 & 1.29 & $58.9^{d}$ \\
\hline & $\begin{array}{l}\text { I am able to calm myself down when I am anxious and } \\
\text { worried about my partner's increased cancer risk }\end{array}$ & 3.84 & 1.18 & $67.4^{d}$ \\
\hline \multicolumn{5}{|l|}{ Couple factors } \\
\hline \multirow[t]{2}{*}{ Supportive role 3} & $\begin{array}{l}\text { I feel confident in my ability to support my partner with her } \\
\text { increased cancer risk }\end{array}$ & 4.28 & 0.91 & $86.3^{c}$ \\
\hline & $\begin{array}{l}\text { I believe my partner is satisfied with my support provision } \\
\text { in regards to her increased cancer risk }\end{array}$ & 3.92 & 0.93 & $75.8^{c}$ \\
\hline \multirow[t]{2}{*}{ Decision making } & $\begin{array}{l}\text { I am involved in all aspects of decision-making in regards to } \\
\text { my partner's increased cancer risk }\end{array}$ & 3.44 & 0.94 & $45.3^{c}$ \\
\hline & $\begin{array}{l}\text { Would you have liked your partner to involve you more in } \\
\text { dealing with her increased cancer risk? }\end{array}$ & .21 & 0.41 & $21.1^{e}$ \\
\hline Perceived coping & $\begin{array}{l}\text { I believe my partner is coping well with her increased } \\
\text { cancer risk }\end{array}$ & 3.75 & 0.89 & $67.4^{c}$ \\
\hline Agreement & $\begin{array}{l}\text { My partner and I agree on most issues regarding breast/ } \\
\text { ovarian cancer }\end{array}$ & 3.87 & 0.75 & $70.5^{c}$ \\
\hline Satisfaction & $\begin{array}{l}\text { Are you satisfied with how your partner has chosen to } \\
\text { manage her increased cancer risk? }\end{array}$ & 1.05 & 0.22 & $94.7^{e}$ \\
\hline Approach & $\begin{array}{l}\text { My partner and I deal with her increased cancer risk as a } \\
\text { team }\end{array}$ & 3.87 & 0.88 & $69.5^{c}$ \\
\hline Communication & $\begin{array}{l}\text { My partner and I communicate openly about all aspects of } \\
\text { being at high risk of developing breast/ovarian cancer }\end{array}$ & 3.89 & 0.92 & $74.7^{c}$ \\
\hline
\end{tabular}

Bracketed items refer to items that were combined to attain a mean score: 1 , mean fatalism; 2 , mean self-regulatory ability; 3 , mean supportive role. ${ }^{a}$ Response options ranged from "much lower" (1) to "much higher" (5), and percentage of partners who selected "a little higher" or "much higher" is reported. "Response options ranged from "never" (1) to "always" (5), and percentage of partners who selected "often" or "always" is reported. "Response options ranged from "strongly disagree" (1) to "strongly agree" (5), and percentage of partners who selected "agree" or "strongly agree" is reported. "Response options ranged from "not at all" (1) to "very much" (5), and percentage of partners who selected "quite a bit" or "very much" are reported. "Response options were "yes" and "no," and percentage of partners who selected "yes" are reported.

The results of the bivariate analyses of individual and couple factors and potentially confounding variables influencing partners' general and cancer-specific distress are shown in Table 3.

From the predictor variables entered into the multivariable analyses (see variables in bold type in Table 3), perceived breast cancer risk, monitoring, and the interaction of perceived breast cancer risk and monitoring remained in the final multinomial logistic regression model for total IES. Greater perceived breast cancer risk predicted greater total intrusive and avoidant ideation for partners $(\beta=0.81$, odds ratio $[\mathrm{OR}]=2.24, P=$ $0.04)$, whereas monitoring style was not associated with total IES scores. The results further demonstrated a significant interaction indicating that there was a moderating effect of monitoring on perceived risk in that greater perceived risk and higher monitoring was associated with lower cancer-related 
Table 3

Bivariate analyses of variables associated with partners' distress levels

\begin{tabular}{|c|c|c|}
\hline Factor & IES & DASS \\
\hline \multicolumn{3}{|l|}{ Individual factors } \\
\hline \multicolumn{3}{|l|}{ Perceived risk } \\
\hline Breast cancer & 0.19 & 0.14 \\
\hline Ovarian cancer & 0.08 & $0.23^{c}$ \\
\hline \multicolumn{3}{|l|}{ Beliefs and expectancies } \\
\hline Screening & 0.05 & -0.12 \\
\hline Prophylactic & -0.04 & 0.13 \\
\hline Mean fatalism & 0.09 & -0.06 \\
\hline Optimism & -0.16 & 0.13 \\
\hline \multicolumn{3}{|l|}{ Self-regulatory strategies } \\
\hline Mean self-regulatory ability & $-0.30^{d}$ & -0.18 \\
\hline \multicolumn{3}{|l|}{ Couple factors } \\
\hline \multicolumn{3}{|l|}{ Supportive role } \\
\hline Mean supportive role & 0.03 & -0.05 \\
\hline \multicolumn{3}{|l|}{ Decision making } \\
\hline Involved all aspects & -0.01 & -0.13 \\
\hline More involvement ${ }^{a}$ & -1.41 & -1.55 \\
\hline Perceived coping & $-0.25^{c}$ & -0.05 \\
\hline Agreement & 0.12 & 0.06 \\
\hline Satisfaction $^{a}$ & -1.53 & -1.45 \\
\hline Approach & 0.02 & -0.11 \\
\hline Communication & -0.06 & 0.18 \\
\hline \multicolumn{3}{|l|}{ Confounding variables } \\
\hline Age & -0.03 & -0.09 \\
\hline Education level & 0.04 & 0.06 \\
\hline Relationship length & -0.04 & -0.11 \\
\hline Having a daughter ${ }^{a}$ & 0.4 & 1.9 \\
\hline No. of FDRs and SDRs ${ }^{b}$ & 0.12 & 0.12 \\
\hline Wife's mutation status ${ }^{a}$ & 1.21 & 0.44 \\
\hline Time since attendance for genetic counseling & -0.16 & -0.05 \\
\hline Cancer-related event ${ }^{a}$ & 0.21 & $2.10^{c}$ \\
\hline Monitoring score & 0.13 & $0.27^{d}$ \\
\hline Breast cancer genetics knowledge score & -0.03 & -0.14 \\
\hline Concerns for children & 0.20 & 0.03 \\
\hline
\end{tabular}

IES, Impact of Events Scale; DASS, Depression, Anxiety, and Stress Scale-Brief version; FDRs, first-degree relatives; SDRs, second-degree relatives.

${ }^{a}$ Variable is categorical, Mann-Whitney $U$ test is used and $z$ value is reported (for all other continuous variables, Spearman's rank correlations are reported).

${ }^{b}$ Wife's total number of first- and second-degree relatives with breast and/or ovarian cancer.

${ }^{c} P<0.05 ;{ }^{d} P<0.01$. Variables in bold type had a significance of $P \leq 0.1$ and were included in the subsequent multivariable analyses.

distress $(\beta=-0.36, \mathrm{OR}=0.70, P=0.01)$. This is a seemingly paradoxical result, which may be the product of the arbitrary categorization of partners' total IES scores (further investigations of this interaction using binary logistic regression with dichotomized, instead of categorized, total IES scores revealed no significant interaction).

Table 4 shows the results of the multivariate analyses for variables predicting partners' total DASS scores. As can be seen, the final ordinal logistic regression model for total DASS included perceived breast cancer risk, monitoring score, cancer-related event, and open communication. Higher perceived breast cancer risk and higher monitoring were associated with higher DASS scores. Also, partners whose wives did not have a breast/ovarian cancer-related event occur in their family in the past year had higher total DASS scores than partners whose wives reported having such an event occur in the past year. Further, open communication was associated with lower total DASS scores for partners.

\section{Association between partners' and women's adjustment}

The means and SDs for partners and women on the IES, DASS, breast/ovarian cancer genetics knowledge scores, and monitoring scores as well as the correlations and results from significance testing comparing partners' and women's scores are summarized in Table 5. The means for partners' DASS Depression, Anxiety, and Stress subscale scores were 5.7 (95\% CI: 4.2-7.2), 3.3 (95\% CI: 2.2-4.4) and 9.1 (95\% CI: 8.6-9.7) respectively, compared with the Australian normative sample (males) of 6.6 (95\% CI: 6.2-7.0), 4.6 (95\% CI: 4.3-4.9) and 9.9 (95\% CI: 9.4-10.4), ${ }^{24}$ which indicates comparable levels of distress. As predicted, Spearman's rank correlations between partners' and women's IES scores revealed significant correlations between partners' and women's IES intrusion $(r=0.29$, $P=0.005)$, and IES avoidance scores $(r=0.30, P=0.004)$, as well as total IES scores $(r=0.29, P=0.005)$. However, partners' and women's scores on the DASS Depression, Anxiety, and Stress subscale, total DASS scores, and breast/ovarian cancer genetics knowledge scores did not significantly correlate. Eight percent (8.4\%), $4.2 \%$, and $6.4 \%$ of partners had scores indicative of severe to extremely severe levels of depression (score of $\geq 21$ ), anxiety (score of $\geq 15$ ) and stress (score of $\geq 26$ ), respectively. In relation to cancer-specific distress, $4.2 \%$, $11.6 \%$, and $4.2 \%$ of partners had scores on the IES Intrusion and IES Avoidance subscales and total IES scores, respectively, indicative of a significant distress response in relation to their wives being at high risk of developing breast/ovarian cancer (scores $\geq 20$ on each of the subscales and scores $\geq 40$ for total IES).

\section{Information and support needs}

Only $33.7 \%$ of partners reported that they were well informed about breast/ovarian cancer and issues in relation to being at increased risk, and $57.9 \%$ of partners reported that they would like more information about breast/ovarian cancer. The majority of partners $(78.9 \%)$ reported that they were coping well with their wife's increased cancer risk, and 32.6\% reported that they would like more support in dealing with their wife's risk. Twenty-five percent (25.3\%) of partners had suggestions for improving services for partners of high-risk women, including provision of information on breast/ovarian 
Table 4

Summary of ordinal logistic regression results for variables predicting general distress

\begin{tabular}{|c|c|c|c|c|c|c|c|}
\hline Variable & $\beta$ & SE & Wald $\chi^{2}$ & $\mathrm{df}$ & OR & $95 \%$ CI & $P$ \\
\hline Perceived breast cancer risk & 0.53 & 0.26 & 4.07 & 1 & 1.69 & $1.02-2.83$ & 0.04 \\
\hline Monitoring score & 0.14 & 0.07 & 3.93 & 1 & 1.15 & $1.00-1.32$ & 0.05 \\
\hline Cancer-related event ${ }^{a}$ & 0.92 & 0.40 & 5.20 & 1 & 2.51 & $1.15-5.50$ & 0.02 \\
\hline Open communication & -0.54 & 0.23 & 5.68 & 1 & 0.58 & $0.37-0.91$ & 0.02 \\
\hline
\end{tabular}

Model $\chi^{2}(4)=17.16, P=0.002$. OR, odds ratio; CI, confidence interval.

${ }^{a}$ Comparison category is presence of a cancer-related event.

Table 5

Partners' and women's scores on outcome and covariate variables

\begin{tabular}{|c|c|c|c|c|c|c|}
\hline \multirow[b]{2}{*}{ Scale } & \multicolumn{2}{|c|}{$\begin{array}{l}\text { Partners } \\
(\mathrm{N}=95)\end{array}$} & \multicolumn{2}{|c|}{$\begin{array}{l}\text { Women } \\
(\mathrm{N}=95)\end{array}$} & \multirow{2}{*}{$\begin{array}{c}\text { Correlation } \\
\text { of partners' } \\
\text { and women's } \\
\text { scores }\end{array}$} & \multirow{2}{*}{$\begin{array}{l}\text { Comparison } \\
\text { of partners' } \\
\text { and women' } \\
\text { scores } \\
Z^{a}\end{array}$} \\
\hline & Mean & $\mathrm{SD}$ & Mean & $\mathrm{SD}$ & & \\
\hline Total IES & 10.5 & 13.3 & 16.3 & 15.3 & $0.29^{c}$ & $-3.12^{c}$ \\
\hline Intrusion & 4.4 & 6.5 & 7.3 & 7.9 & $0.29^{c}$ & $-2.92^{c}$ \\
\hline Avoidance & 6.1 & 8.3 & 9.1 & 8.5 & $0.30^{c}$ & $-3.01^{c}$ \\
\hline Total DASS & 18.2 & 20.3 & 21.6 & 21.3 & 0.02 & -0.83 \\
\hline Depression & 5.7 & 7.6 & 5.2 & 6.9 & 0.05 & -0.43 \\
\hline Anxiety & 3.3 & 5.4 & 3.8 & 6.0 & 0.03 & -0.44 \\
\hline Stress & 9.1 & 9.2 & 11.7 & 10.1 & -0.01 & -1.63 \\
\hline \multicolumn{7}{|c|}{$\begin{array}{l}\text { Breast/ovarian } \\
\text { Cancer Genetics } \\
\text { Knowledge Score }\end{array}$} \\
\hline & 3.1 & 1.5 & 3.8 & 1.1 & 0.15 & $3.23^{c}$ \\
\hline Monitoring Score & 9.0 & 2.9 & 10.0 & 2.7 & 0.10 & 1.86 \\
\hline
\end{tabular}

${ }^{a}$ Wilcoxon test performed for scores with a skewed distribution.

${ }^{b}$ Paired $t$ test performed for normally distributed scores.

${ }^{c} P<0.01$.

cancer and risk management options in the form of a newsletter, seminar, or Web site; strategies on how to best support their wife; greater encouragement from health professionals for partners to attend women's appointments; and meeting with other partners, in particular partners whose wives had undergone prophylactic surgery to find out what to expect after the surgery.

\section{Impact of wife's increased cancer risk on the couples' relationship}

Approximately half of the partners (49.5\%) reported that they often worried about their children having inherited their wife's increased cancer risk. Significantly more partners with daughters $(z=-2.04, P<0.05)$ reported that they worried about their children being at risk than partners who did not have female offspring. In terms of the impact of women's increased breast/ovarian cancer risk on the couple's relationship, $70.5 \%$ of partners reported no impact on their relationship. Some partners (17.9\%) reported that their wife's high risk had a positive impact on their relationship in that it had brought them closer together, they communicated more often and openly about her risk, and they concentrated on enjoying their time together more. In contrast, $11.6 \%$ of partners reported that their wife's risk had a negative impact on their relationship due to the anxiety and depression experienced by their wife and themselves about the increased risk, worry about children also being at increased risk, and the negative physical and psychological impacts of prophylactic surgery such as early menopausal symptoms and lack of interest in sex.

\section{DISCUSSION}

To date, the current study is the first to investigate the psychological adjustment of partners of female BRCA1/2 mutation carriers and women with unknown mutation status at high risk of developing breast/ovarian cancer. This study revealed that a small proportion of partners of women at high risk of developing breast/ovarian cancer are experiencing elevated levels of distress necessitating clinical interventions. In comparison with previous studies, the partners in the current sample had higher total intrusion and avoidance scores (mean total IES score of 10.5) than partners of mutation carriers (mean total IES score of 5.8$)^{2}$ and higher mean intrusion (mean score of 4.4) and mean avoidance (mean score of 6.1) scores than partners of high-risk women applying for genetic testing (1.4 and 1.3, respectively). ${ }^{9}$ In contrast, the study of Metcalfe et al. ${ }^{8}$ that included only partners of mutation carriers found higher mean intrusion (7.4) and avoidance (7.3) scores as compared with the partners in the current sample, which included just a small number of partners of mutation carriers. These latter findings are also consistent with the findings of our previous qualitative study regarding partners of mutation carriers. It is possible that partners of mutation carriers experience greater cancer-specific distress than partners of at-risk women with unknown mutation status. In this current study, we found no association between carrier status and total intrusion and avoidance scores; however, the small number of carriers included $(n=13$, $13.7 \%$ ) may have limited our ability to detect any differences. Interestingly, partners in the current study scored higher on the Intrusion and Avoidance subscales than men who themselves are at high risk of being BRCA1/2 mutation carriers (2.7 and 1.7, respectively), ${ }^{2}$ and scored higher on the Avoidance subscale than men at increased risk of prostate cancer (4.4). ${ }^{26}$

Although the assessment of general distress revealed that the current sample had levels of general depression, anxiety, 
and stress similar to those reported for the Australian normative sample ${ }^{24}$ for all three subscales, we identified a subgroup of men with significant general distress responses. The predictors of general distress included factors specifically related to their wives' increased risk, suggesting that these factors may be influencing this subgroup more than those with normal levels of distress. These breast cancer risk-specific factors may uniquely contribute to distress levels in this population rather than representing a generic form of distress, a fact that is also underscored by the high levels of cancer-specific distress.

As predicted by the C-SHIP model, partners' higher perceived risk of breast cancer for their wives was found to be predictive of higher cancer-specific and general distress for partners in this study. This is consistent with a large body of literature (e.g., see Thewes et al. ${ }^{22}$ ) on the impact of elevated perceived risk on distress among women at increased risk of hereditary breast/ovarian cancer. Also consistent with the CSHIP model, monitoring attentional style was found to be predictive of partners' general distress, which is consistent with previous research showing that high monitoring is associated with elevated states of arousal and greater psychological distress. ${ }^{27-29}$ This is thought to be due to high monitors' pervasive focus on threat and danger and higher levels of perceived vulnerability, which activates distress and a state of prolonged arousal in these individuals. ${ }^{29} \mathrm{We}$ also found an interaction that does not fit the C-SHIP model, indicating that there is a moderating effect of monitoring on perceived risk in that greater perceived risk and higher monitoring is associated with lower cancer-related distress. Although the validity of this finding requires further investigation, this seemingly paradoxical result may be explained by the cognitive dissonance theory ${ }^{30}$ because they see themselves at high risk and characteristically engage in health-related information-seeking behaviors, high monitoring individuals feel that their actions are coherent with these schemata, leading to reduced distress. High monitors who perceive lower risk may have higher distress levels because receiving information about their increased risk at a risk assessment clinic is less consistent with their cognitive schema.

We found that partners who reported communicating openly with their wives regarding her increased cancer risk had lower levels of distress. This finding is consistent with the qualitative findings of our previous study, ${ }^{11}$ and another survey study, that investigated the role of relationship communication on the distress levels of women undergoing genetic testing and their partners and found that greater comfort sharing concerns about the genetic testing experience at baseline was associated with lower levels of cancer-specific distress 6 months post-testing among partners. ${ }^{5}$

We additionally found that the occurrence of a breast/ovarian cancer-related event in the women's family during the past year was associated with lower levels of general distress for partners. It may be that vicariously experiencing such an event provides these partners with the opportunity to work through their feelings and develop relevant coping skills resulting in better adjustment. By contrast, women at increased risk of developing hereditary breast cancer who had experienced a breast cancer-related event in their family in the past year have been found to be more likely to have high levels of psychological distress. ${ }^{31}$ It is possible that experiencing such an event has a differential effect for women and for their partners. Further research is required to determine whether the positive association between experiencing a recent cancer-related event in the family and better adjustment for partners can be replicated and to identify possible reasons for this relationship.

Two aspects of the C-SHIP model were not confirmed by the findings, in particular no multivariable associations were found between distress and (i) positive beliefs about the onset and management of breast/ovarian cancer and (ii) greater selfregulatory ability. Furthermore, several of the couple factors hypothesized to correlate with psychological adjustment based on findings from the qualitative phase of this research were not found to be significant (e.g., partners feeling adequate in providing support to their wives, greater involvement in decisionmaking). The items measuring the hypothesized factors were designed specifically for the study and were not validated measures of these concepts. Hence, the items used in this study may not have appropriately measured these factors, and future research is needed to develop validated measures of these constructs. Another reason why the results of this quantitative phase did not reflect the findings in the pilot qualitative phase may be that some of these specific couple factors (e.g., team versus independent approach, supportive role) are not amenable to being assessed by Likert scale questions in self-administered questionnaires. Conversely, the hypotheses for these couple factors were based on the major themes identified in the interviews. Although these identified factors may be relevant in describing partners' experiences, it is possible that these factors do not have a simple relationship with partners' adjustment and are indicative of other more complex factors that were not identified by the analyses of the interviews.

\section{Limitations}

This was a single-assessment study design that precluded the possibility of obtaining information about causality. Whether partners had attended the genetic counseling appointments was not controlled for in this study, which may influence partners' adjustment. Also, although we asked partners and wives to complete the study instruments separately, there was no way to confirm that this actually happened. The sample size was relatively small, and this study should be replicated with a larger sample. Some of the factors predictive of partners' adjustment approached but failed to reach significance, and a larger sample would have greater power to detect smaller effect sizes. Moreover, the sample did not include an adequate number of partners of mutation carriers and women who had undergone prophylactic surgery to enable between-group comparisons of psychological adjustment. In addition, no information is available about individuals who declined participation, and it may be that partners who participated were self-selected and differed in their levels of psychological distress and coping from part- 
ners who did not participate in this study. Finally, participants in this study tended to have higher education levels than the general Australian population, with $65 \%$ of partners having a postschool qualification compared with $46 \%$ of people in the general population ${ }^{32}$; however, individuals attending familial cancer clinics have been found to have an above-average educational level, and hence our data are generalizable to those attending clinics.

\section{Clinical implications}

Thus far, research has almost exclusively focused on identifying high-risk women most vulnerable to psychological distress. However, women in partnered relationships need to be considered as being part of a larger system, and it is important to keep in mind that they are not being affected by their cancer risk in isolation. In light of the findings of this study, we suggest that service delivery and interventions for high-risk women focus not only on the adjustment of women but on that of partners as well as on the couple as a whole. The design of such interventions needs to take into account the reciprocal relationship between the impact of the women's and their partners' distress. Integrating this new perspective into clinical practice may be beneficial for both women and their partners because addressing the needs of partners may lead to better coping for partners, thereby allowing partners to provide more effective support to the women.

Our finding that monitoring attentional style was predictive of higher distress for partners suggests that tailoring of clinical and psychoeducational interventions for partners to their preferred attentional styles of coping may be important. Empirical findings indicate that high monitors benefit most if information is presented in a manner that distances or cools it, so their sense of vulnerability and distress does not panic them, and the personal relevance of the disease is framed less negatively. ${ }^{12}$ In contrast, low monitors fare better with more minimal actionoriented messages, with sufficient opportunity to psychologically tune out from what is facing them. ${ }^{12}$ Drawing on recommendations made for high monitoring at-risk women, ${ }^{12,33}$ high monitoring partners may need to be reassured in a reasonable manner (e.g., about the high potential for cure after early detection) to undercut their catastrophic thinking and be provided with accurate procedural information to enable them step-by-step preparation for the management of their wives' risk (e.g., information regarding screening frequency and prophylactic surgery); conversely, low monitoring partners may need interventions that emphasize and facilitate understanding of their wives' risk as well as ways to manage cancer-related distress.

The majority (58\%) of men in our sample reported that they would like more information about breast/ovarian cancer. Considering the influential role that partners were found to play in women's decision making in both current and previous research, ${ }^{4}$ meeting men's information needs appears important. These findings suggest that partners of at-risk women may benefit from information and support services specifically designed and tailored for them. These results also underscore the benefits of health professionals' educating partnered women in the benefits of bringing their partners along to the consultation.

Our findings suggest that it may be helpful for health professionals to promote having a team approach in dealing with this health stressor to ease the burden on the women, given that having a team approach has been found to be beneficial for couples dealing with cancer. ${ }^{34,35}$ Couples who are experiencing difficulties in adjusting to women's high cancer risk may benefit from interventions similar to those designed for couples dealing with a diagnosis of breast cancer, ${ }^{34}$ which helps couples define the women's high risk as "our problem" and encourage couples to create a meaningful structure that provides direction for their coping efforts. Again, similar to couples dealing with women's breast cancer diagnosis, ${ }^{36}$ group couple therapy may aid in couples' adjustment if they are experiencing communication difficulties in relation to the women's increased risk.

\section{ACKNOWLEDGMENTS}

The authors are very grateful to our participants for their willingness to take part in this study. They also thank Dr. Nadine Kasparian for assistance with statistical analyses and Jennifer Irle for administrative support. Dr. Meiser is supported by a Career Development Award from The National Health and Medical Research Council of Australia (ID 350989).

\section{References}

1. Antoniou A, Pharoah PDP, Narod SA, et al. Average risks of breast and ovarian cancer associated with BRCA1 or BRCA2 mutations detected in case series unselected for family history: A combined analysis of 22 studies. Am J Hum Genet 2003;72:1117-1130.

2. Lodder L, Frets PG, Trijsburg RW, Meijers-Heijboer EJ, et al. Psychological impact of receiving a BRCA1/BRCA2 test result. Am J Med Genet 2001;98:15-24.

3. Lerman C. Mammography adherence and psychological distress among women at risk for breast cancer. J Natl Cancer Inst 1993;85:1074-1080.

4. Coyne JC, Anderson KK. Marital status, marital satisfaction, and support processes among women at high risk for breast cancer. J Fam Psychol 1999;13:629-641.

5. Manne SL, Audrain J, Schwartz MD, Main D, et al. Associations between relationship support and psychological reactions of participants and partners to BRCA1 and BRCA2 testing in a clinic-based sample. Ann Behav Med 2004;28:211-225.

6. Botkin J, Smith K, Croyle R, Baty BJ, et al. Genetic testing for a BRCA1 mutation: Prophylactic surgery and screening behavior in women 2 years post testing. Am J Med Genet 2003;118A:201-209.

7. Lodder LN, Frets PG, Trijsburg RW, Meijers-Heijboer EJ, et al. Presymptomatic testing for BRCA1 and BRCA2: how distressing are the pre-test weeks? J Med Genet 1999;36:906-913.

8. Metcalfe KA, Liede A, Trinkaus M, Hanna D, et al. Evaluation of the needs of spouses of female carriers of mutation in BRCA1 and BRCA2. Clin Genet 2002;62: 464-469.

9. Dudok de Wit AC, Tibben A, Duivenvoorden HJ, Frets PG, et al. Psychological distress in applicants for predictive DNA testing for autosomal dominant, heritable, late onset disorders. J Med Genet 1997;34:382-390.

10. Lloyd SM, Watson M, Oaker G, Sacks N, et al. Understanding the experience of prophylactic bilateral mastectomy: a qualitative study of ten women. Psychooncology 2000;9:473-485.

11. Mireskandari S, Meiser B, Sherman K, Warner BJ, et al. Evaluation of the needs and concerns of partners of women at high risk of developing breast/ovarian cancer. Psycho-Oncol 2006;15:96-108.

12. Miller SM, Shoda YHurley K, et al. Applying cognitive-social theory to healthprotective behavior: breast self-examination in cancer screening. Psychol Bull 1996; 119:70-94.

13. Ben-Zur H, Gilbar O, Lev S. Coping with breast cancer: Patient, spouse, and dyad models. Psychosom Med 2001;63:32-39.

14. Hoskins CN. Adjustment to breast cancer in couples. Psychol Rep 1995;77:435-454. 


\section{Mireskandari et al.}

15. Northouse LL, Dorris G, Charron-Moore C. Factors affecting couples' adjustment to recurrent breast cancer. Soc Sci Med 1995;41:69-76.

16. Australian Cancer Network. Guidelines on familial aspects of cancer. Sydney: Australian Cancer Network, 1999.

17. Nathanson CA. Sex roles as variables in preventive health behavior. J Community Health 1977;3:142-155.

18. National Health \& Medical Research Council (NHMRC) National Breast Cancer Centre Psychosocial Working Group. Psychosocial clinical practice guidelines: providing information, support, and counseling for women with breast cancer. Canberra ACT: Commonwealth of Australia, 2000.

19. Miller SM. Monitoring and blunting: validation of a questionnaire to assess styles of information seeking under threat. J Pers Soc Psychol 1987;52:345-353.

20. Miller SM, Roussi P, Caputo GC, Kurus L. Patterns of children's coping with an aversive dental treatment. Health Psychol 1995;14:236-246.

21. Meiser B, Butow PN, Barratt A, Gattas M, et al. Risk perception and knowledge of breast cancer genetics in women at increased risk of developing hereditary breast cancer. Psychol Health 2001;16:297-311.

22. Thewes B, Meiser B, Tucker K, Schnieden V. Screening for psychological distress in women at increased risk of developing breast cancer: a review of literature. Psychol Health Med 2003;8:289-304.

23. Van Roosmalen M, Stalmeier P, Verhoef L, Hoekstra-Weebers JEHM, et al. Impact of BRCA1/2 testing and disclosure of a positive result on women affected and unaffected with breast or ovarian cancer. Am J Med Genet 2004;124A:346-355.

24. Lovibond SH, Lovibond PF, et al. Manual for the depression, anxiety, and stress scales, 2nd ed. Sydney: The Psychology Foundation of Australia Inc., 1995.

25. Horowitz M, Wilner N, Alvarez W. The Impact of Event Scale: a measure of subjective stress. Psychosom Med 1979;41:209-218.
26. Bratt O, Damber J-E Emanuelsson M, Kristofferssen U, et al. Risk perception, screening practice and interest in genetic testing among unaffected men in families with hereditary prostate cancer. Eur J Cancer 2000;36:235-241.

27. Tercyak KP, Lerman C, Peshkin BN, Hughes C,et al. Effect of coping style and BRCA1 and BRCA2 test results on anxiety among women participating in genetic counseling and testing for breast and ovarian cancer risk. Health Psychol 2001;20:217-222.

28. Miller S, Roussi P, Altman D, Helm W, et al Effects of coping style on psychological reactions of low-income, minority women to colposcopy. J Reprod Med 1994;39:711718.

29. Miller S, Mangan C. Interacting effects of information and coping style in adapting to gynecologic stress: should the doctor tell all? J Pers Soc Psychol 1983;45:223-236.

30. Elliot A, Devine P. On the motivational nature of cognitive dissonance: dissonance as psychological discomfort. J Pers Soc Psychol 1994;67:382-394.

31. Meiser B, Butow PN, Schnieden V, Gattas M, et al. Psychological adjustment of women at increased risk of developing hereditary breast cancer. Psychol Health Med 2000;5:377-388.

32. Australian Bureau of Statistics. Census expanded community profile. Canberra: Australian Bureau of Statistics, 2001.

33. Fang C, Miller S, Daly M, Hurley K. The influence of attentional style and risk perceptions on intentions to undergo prophylactic oophorectomy among first-degree relatives. Psychol Health 2002;17:365-376.

34. Skerrett K. Couple dialogues with illness: expanding the "we". Fam Syst Health 2003;21:69-80.

35. Persson L, Rasmusson M, Hallberg IR. Spouses' view during their partners' illness and treatment. Cancer Nurs 1998;21:97-105.

36. Katz C, Bosch G, Herzog RE. Group-therapy of couples after breast cancer diagnosis: experimental pilot study. Anticancer Res 1998;18:2267-2270. 